Musées, Patrimoine et Culture scientifiques et techniques

$162 \mid 2015$

novembre-décembre 2015

\title{
20 ans de numérique dans les musées : entre monstration et effacement
}

Daniel Schmitt et Muriel Meyer-Chemenska

\section{OpenEdition \\ Journals}

Édition électronique

URL : http://journals.openedition.org/ocim/1605

DOI : 10.4000/ocim.1605

ISSN : 2108-646X

Éditeur

OCIM

Édition imprimée

Date de publication : 1 novembre 2015

Pagination : $53-57$

ISSN : 0994-1908

Référence électronique

Daniel Schmitt et Muriel Meyer-Chemenska, « 20 ans de numérique dans les musées : entre

monstration et effacement », La Lettre de I'OCIM [En ligne], 162 | 2015, mis en ligne le 18 janvier 2016, consulté le 14 novembre 2019. URL : http://journals.openedition.org/ocim/1605 ; DOI : 10.4000/ocim. 1605

Ce document a été généré automatiquement le 14 novembre 2019.

Tous droits réservés 


\title{
20 ans de numérique dans les musées : entre monstration et effacement
}

\author{
Daniel Schmitt et Muriel Meyer-Chemenska
}

Mémoires d'Egypte en 1990 (Strasbourg, Paris, Berlin) est l'une des premières expositions immersives à associer étroitement les œuvres et les médias. Le parcours nécessite un casque à réception infra-rouge.

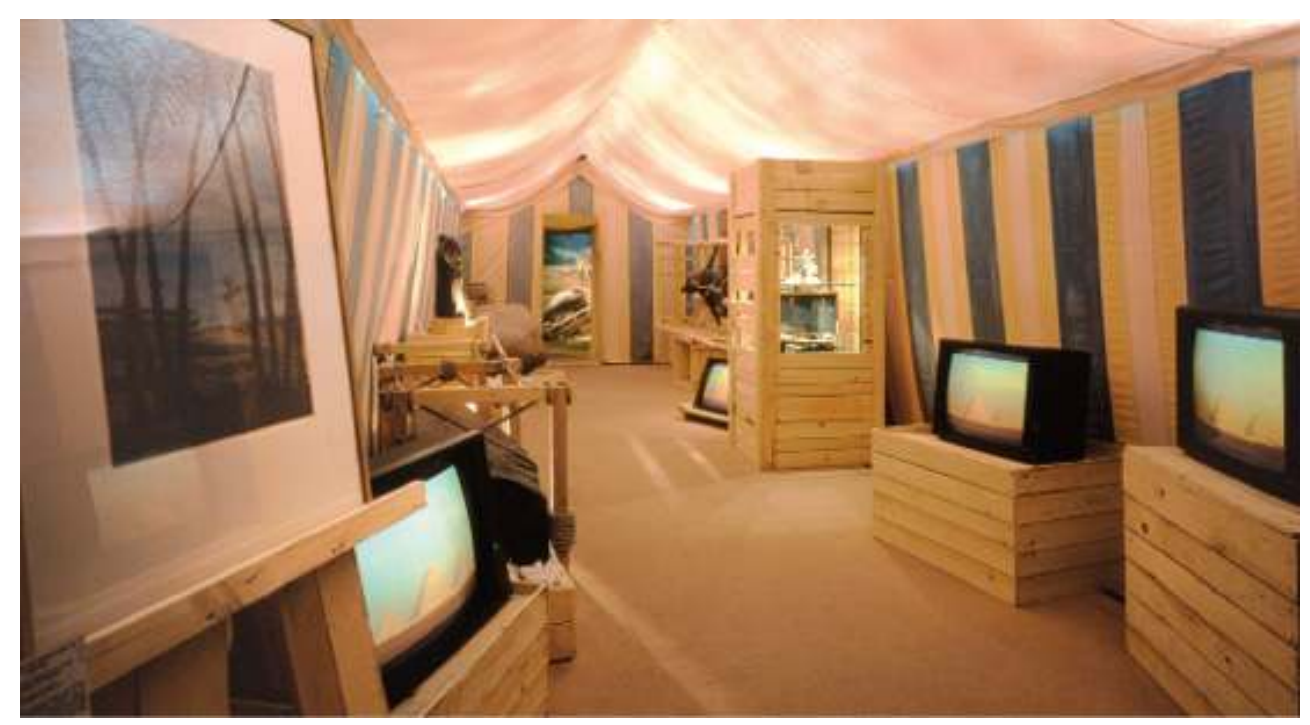

(c) Daniel Schmitt

1 Les outils numériques d'aide à la visite, sites Internet, bornes multimédia, PDA et tablettes tactiles s'invitent depuis une vingtaine d'années dans les expositions permanentes et temporaires. Et ce faisant, ils participent d'une transformation durable et profonde des pratiques des musées et des visiteurs. Remarquons que parmi les nouvelles pratiques, l'interactivité, si souvent mise en avant, n'est pas exclusive au numérique. Bien avant l'ère des ordinateurs, des institutions anciennes comme le 
Science Museum de Londres (1852) ou encore le Deutsches Museum à Munich ou plus récemment le Palais de la Découverte et l'Exploratorium de San Francisco proposaient déjà à leurs visiteurs des dispositifs interactifs, des manipulations, des répliques à toucher, des jeux... Le numérique entrant dans les musées a d'abord servi à l'informatisation des collections avant de servir à la visite elle-même. C'est à partir de ces ensembles documentaires que l'on a envisagé à la fin des années 1980 d'offrir aux visiteurs des accès aux collections informatisées (banques $\mathrm{d} \&$ apos ;images et de fiches informatisées du patrimoine) (Andreacola, 2014).

\section{L'écran vidéo, paria de l'exposition}

2 Avant que l'on ne parle de "médiation ", la diffusion instrumentée des contenus scientifiques dans les espaces muséographiques était prise en charge par les audioguides à cassette analogique. Le défilement physique de la bande magnétique dictait le rythme de la visite plus qu'il ne l'accompagnait. Dans le même temps, la diffusion de films vidéo à l'aide de lecteurs analogiques se faisait dans des espaces dédiés. À la fois pour des raisons techniques (format de production ${ }^{1}$, pilotage, maintenance, son ouvert qui « pollue ») et pour des raisons idéologiques les musées souhaitent séparer le monde des œuvres de celui de la technologie et maintiennent l'écran vidéo hors du parcours de l'exposition. Ce sont des expositions comme Cités-Cinés en 1987 ou Mémoires d'Égypte en 1990 qui consacrent les premières expérimentations autour d'une muséographie que l'on qualifierait aujourd'hui d'immersive et innovante. La scénographie intègre de grands écrans, l'image est diffusée par Laserdisc, un système hybride analogiquenumérique, tandis que le son est diffusé individuellement à l'aide de casques audio à réception infrarouge. Cette technologie permet au visiteur d'entendre la partie audio lorsqu'il se trouve dans une zone de réception et de découvrir une nouvelle liberté: celle de « zapper » en marchant.

3 Ce type de diffusion marque un tournant dans l'utilisation de l'image et du son dans les expositions parce que ces dispositifs ne proposent plus de simples compléments à la visite, mais ils constituent le cœur même d'un dispositif narratif qui nourrit et structure fortement l'expérience des visiteurs. Ces expositions temporaires et itinérantes ont affiché des chiffres exceptionnels de fréquentation ${ }^{2}$. La communauté muséale est partagée. Certains y voient une dérive de l'exposition vers le spectacle et une intrusion coupable de la technique dans le monde de l'art, d'autres au contraire perçoivent les médias comme un moyen d'enrichir les expositions et d'accueillir de nouveaux publics. Mais la critique commune faite à ces dispositifs concerne l'accès au « message » : celui-ci est obligatoire, on capte et on entend les commentaires sonores dès que l'on entre dans une zone de réception. Le visiteur est captif et pour échapper aux commentaires, il faut enlever son casque audio ou changer d'espace. Or beaucoup de responsables souhaitent un accès volontaire, un déclenchement à la demande du visiteur. C'est cette préoccupation qui oriente les premiers dispositifs diffusant du son à partir de mémoires Flash (mémoire non volatile) : avoir un accès immédiat au message que l'on choisit d'écouter. Dans leur forme première, on trouve la rampe Influx ${ }^{\oplus}$ développée en 1995 par les sociétés Créamuse et RSF pour le Neanderthal Museum à Mettman. Installé dans l'exposition permanente, ce dispositif contient toute la médiation sonore. Le visiteur s'y connecte avec la prise d'un casque audio et ce geste déclenche l'accès aux commentaires et à la bande son des films. Cet usage du 
numérique est perçu comme une innovation technologique qui pallie la lecture linéaire des bandes vidéos et leur usure, tout en permettant d'utiliser plusieurs canaux son pour la diffusion multilingue et en offrant une autonomie et une liberté d'accès aux visiteurs. Les premiers audio-guides numériques des années 1997 en sont le principe miniaturisé et mobile. Le numérique n'a donc pas fait irruption du jour au lendemain dans les musées, il a commencé par remplacer les supports traditionnels analogiques de diffusion, sans pour autant modifier radicalement l'expérience de visite. Ce qui constitue une véritable nouveauté, c'est la présence des écrans à proximité des œuvres qui devient acceptable puis légitime.

La rampe Influx ${ }^{\circledR}$ développée en 1995 par les sociétés Créamuse et RSF pour le Neanderthal Museum à Mettman en Allemagne. Elle contient les fichiers numériques des commentaires et la bande son des films. On accède à ces commentaires « sur demande " en introduisant la fiche de son casque dans la rampe.

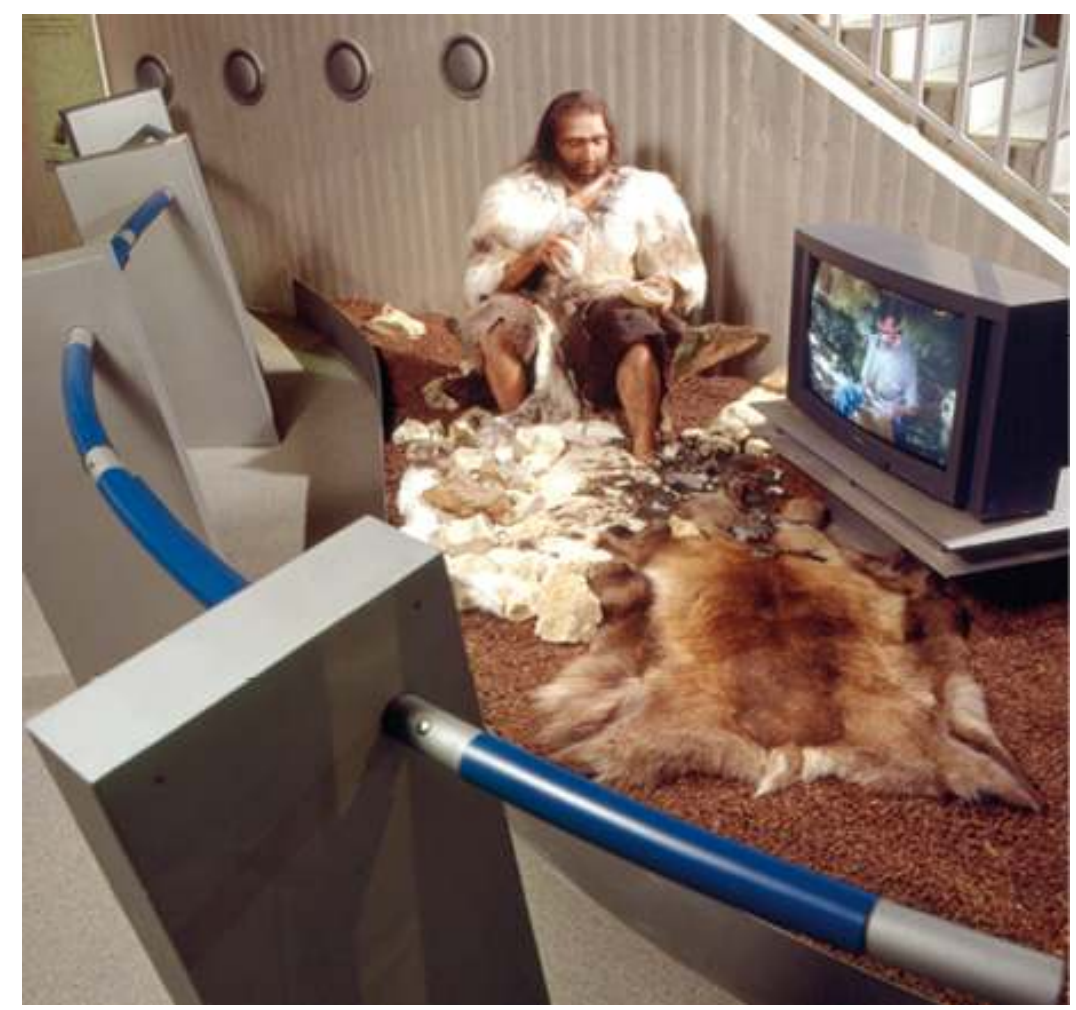

(c) Christian Creutz 
Les premières bornes tactiles installées dans le Neanderthal Museum en 1997. Les musées portent un regard bienveillant sur ces dispositifs qui sont plutôt silencieux et qui permettent de proposer des grandes quantités d'information

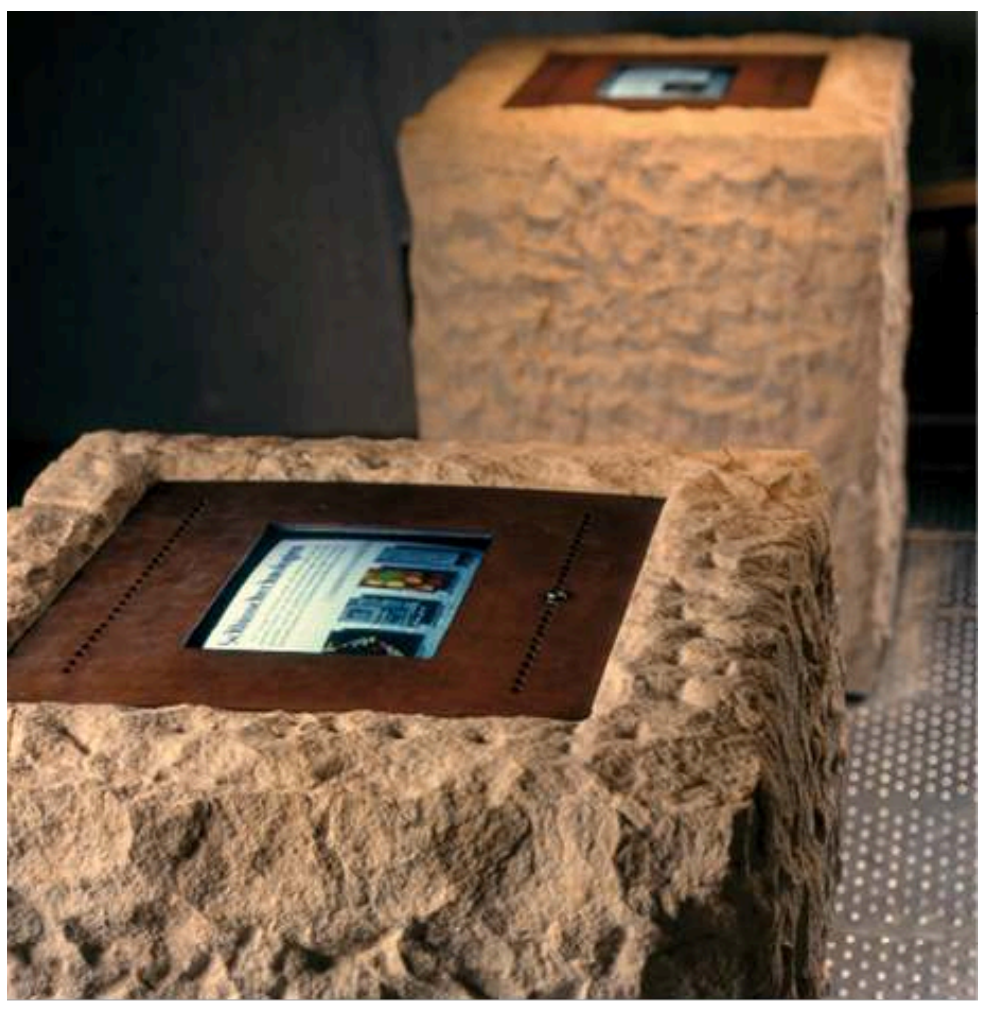

(c) Christian Creutz

\section{L'écran tactile, un invité qui s'impose}

Au milieu des années 1990, l'informatique est encore peu présente dans les foyers et la borne multimédia est une véritable curiosité. Dans le même temps, l'écran devenu tactile s'impose d'autant plus aisément qu'il allège le dispositif interactif et le rend même attractif par sa modernité. Les musées portent un regard bienveillant sur ces «bornes tactiles» peu invasives (peu de bruit et mobiles) qui permettent de proposer des grandes quantités d'information. Équiper son musée de "bornes multimédia " devient un phénomène à la mode et le succès des consultations dépasse de loin les attentes. On pense alors que les visiteurs «touchent l'information du doigt » et qu'il s'agit d'une nouvelle approche cognitive et corporelle de la communication. À vrai dire, la borne multimédia fut d'abord le lieu de la nouveauté où l'on entreposait de nombreux contenus que l'on ne savait pas placer autrement, mais qu'il était bon de stocker quelque part pour les visiteurs. De nombreux contenus étaient là, présents dans la borne-totem des savoirs, même si en fin de compte, il devenait laborieux, pour ne pas dire impossible de les consulter, tant la technologie tactile était balbutiante et la navigation une course d'obstacles. 


\section{Le numérique, entre monstration et effacement}

5 Depuis, la taille, la définition, la restitution des couleurs et la lisibilité de ces écrans ont évolué de façon spectaculaire et les outils numériques de médiation se sont définitivement installés dans le paysage muséal. Au point que certains projets inscrivent le principe d'une médiation «numérique» dans leur cahier des charges avant même de connaître l'intention de médiation et les différentes solutions numériques ou non - qui pourraient être proposées. La médiation numérique rassemble tous les acteurs sous la même bannière de la modernité qu'il est interdit de questionner. Le musée, les visiteurs, le maître d'ouvrage, le territoire, ses habitants... tous entrent soudainement dans l'ère du musée du XXI ${ }^{e}$ siècle, dessinant des futurs enchanteurs.

6 Cette course à la modernité, à l'originalité et à la distinction engage les musées à proposer les derniers dispositifs et le musée est devenu un formidable terrain d'expérimentation de l'innovation numérique. À côté des assistants personnels de visite, nous avons croisé les manipulations RFID, les technologies mobiles, le son hyperdirectionnel, les représentations 3D, 4D, la géolocalisation, la réalité augmentée, les expériences d'interactions gestuelles avec des interfaces 3D relief, les expériences collaboratives Web 2.0, les lunettes intelligentes qui reconnaissent les œuvres et en proposent un commentaire tout en guidant le regard et bien sûr, d'innombrables applications.

Projection 3D relief sans lunettes (Cliffs of Moher Visitor Centre, Irlande, 2015). La projection tente de faire vivre une expérience de découverte du site en survolant les falaises comme un oiseau ou en nageant comme un poisson. Mais la technologie montre vite ses limites quand elle tente de rivaliser avec la réalité, souvent plus simple et plus émouvante.

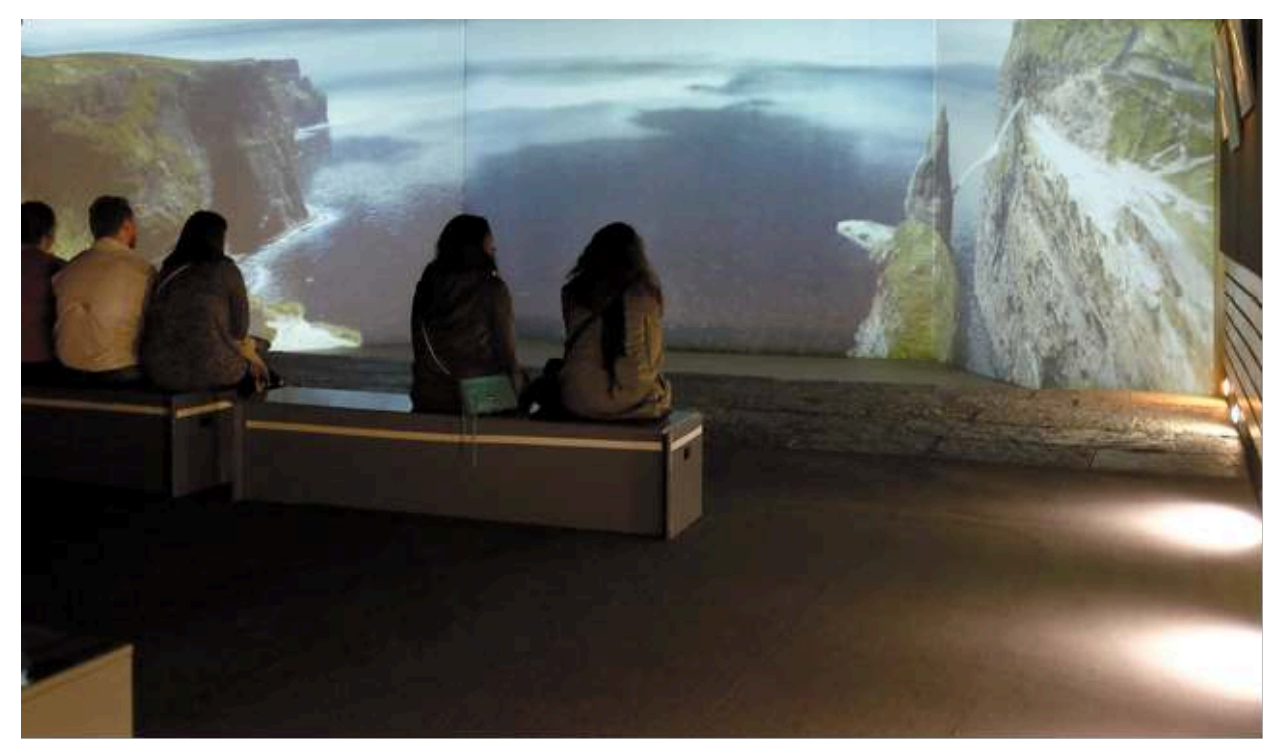

(C) Daniel Schmitt

7 Pour chacun d'eux, le visiteur doit à chaque fois réapprendre l'ergonomie et la navigation qui mettent à l'épreuve ses capacités d'utilisateur. Au mieux, le dispositif constitue une intrigue à laquelle le visiteur trouve une réponse qui lui convient. Au pire il reste une énigme et le visiteur entre dans un labyrinthe séquentiel contraignant qui 
peut absorber toute son énergie, avant même qu'il n'arrive à entrevoir le début de ce qu'il espérait trouver.

8 Ainsi au musée de la Résistance de Bondues, ce sont les guides qui tiennent à la main une tablette tactile. Ils manipulent le dispositif et convoquent à intervalles réguliers les narrations filmées de témoins ayant été en rapport direct avec les expôts. L'avantage est immédiat, les visiteurs ne mettent pas à l'épreuve leurs compétences techniques et se concentrent sur l'histoire de la résistance et sur les objets présentés et racontés (Gellereau et al., 2015).

9 C'est quand les dispositifs apparaissent banals qu'ils deviennent de véritables outils que l'on peut s'approprier: ils apparaissent en quelque sorte transparents, ils «disparaissent " pour que l'on puisse s'en servir sans y penser afin d'établir nos liaisons propres au monde, et in fine augmenter la richesse et la variété des connaissances construites. C'est à la technologie numérique de se modeler aux utilisateurs et non l'inverse. Expérimentés avec un public jeune et conçus du point de vue des utilisateurs, les programmes numériques sur tablettes permettent même de favoriser l'autonomie, la concentration, la satisfaction et l'expérience d'un nouveau rapport au musée des enfants (Bougenies et al., à paraitre 2015).

Or précisément, à peine deviennent-ils ordinaires que ces dispositifs numériques se métamorphosent en innovations technologiques appelées à fasciner pour elles-mêmes en tant qu'objet. Le numérique comme argument principal d'une muséographie innovante doit se montrer, mais en faisant cela, on empêche justement ces dispositifs de devenir transparents c\&apos ;est-à-dire d'entrer dans "un processus d'intégration progressive au monde propre, au corps propre et à la culture propre de l'acteur» (Poizat, 2014). À travers la monstration de la technologie, les dispositifs numériques imposent sans cesse aux visiteurs de revoir leurs habitudes ergonomiques et leurs compétences techniques. Ce faisant, ils font parfois subir aux visiteurs une certaine forme de violence. 
L'audioguide devient transparent au fur et à mesure qu'il se banalise. II cohabite avec la fiche de salle pour proposer différentes modalités de relations entre les visiteurs et les œuvres (musée de l'CEuvre Notre-Dame, Strasbourg, 2014).

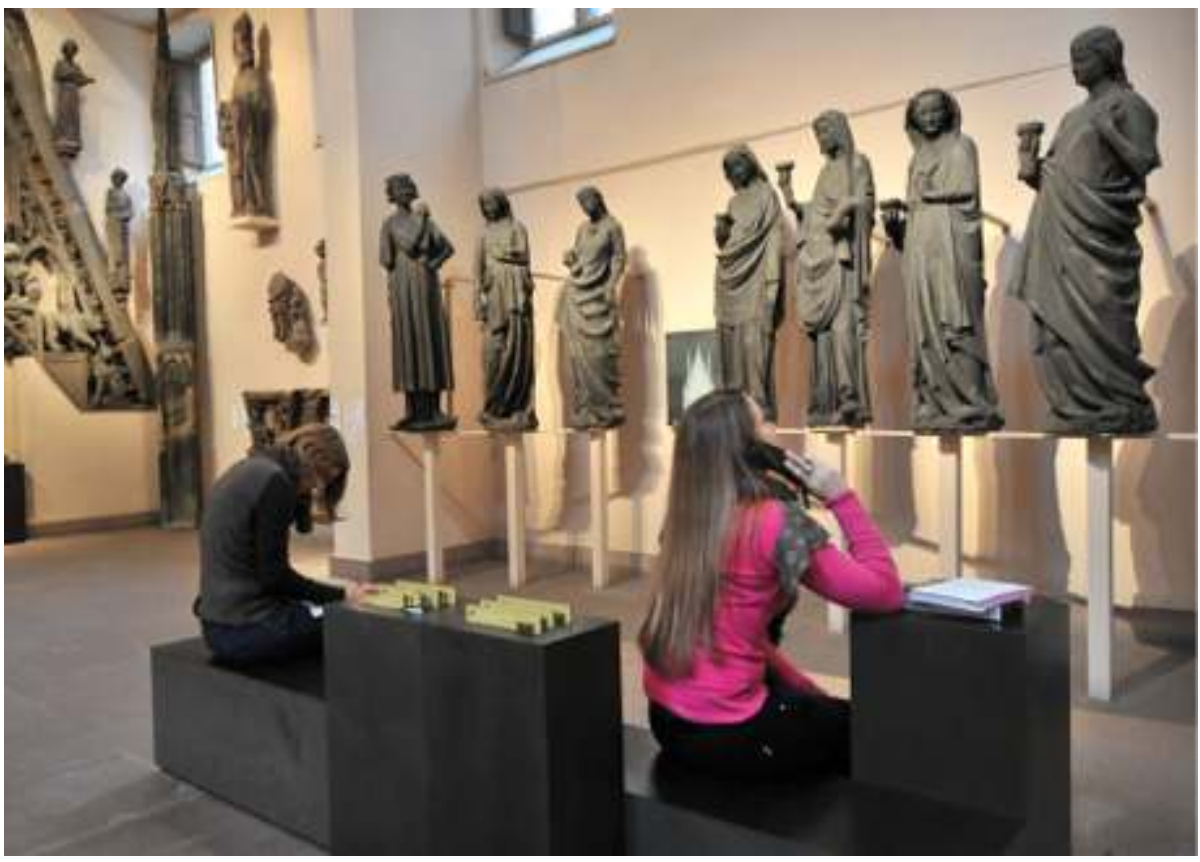

(c) Daniel Schmitt

L'usage des tablettes numériques est devenu ordinaire avec la diffusion des smartphones. Exposition Open Museum \#2 Donald (Palais des Beaux-Arts de Lille, 2015).

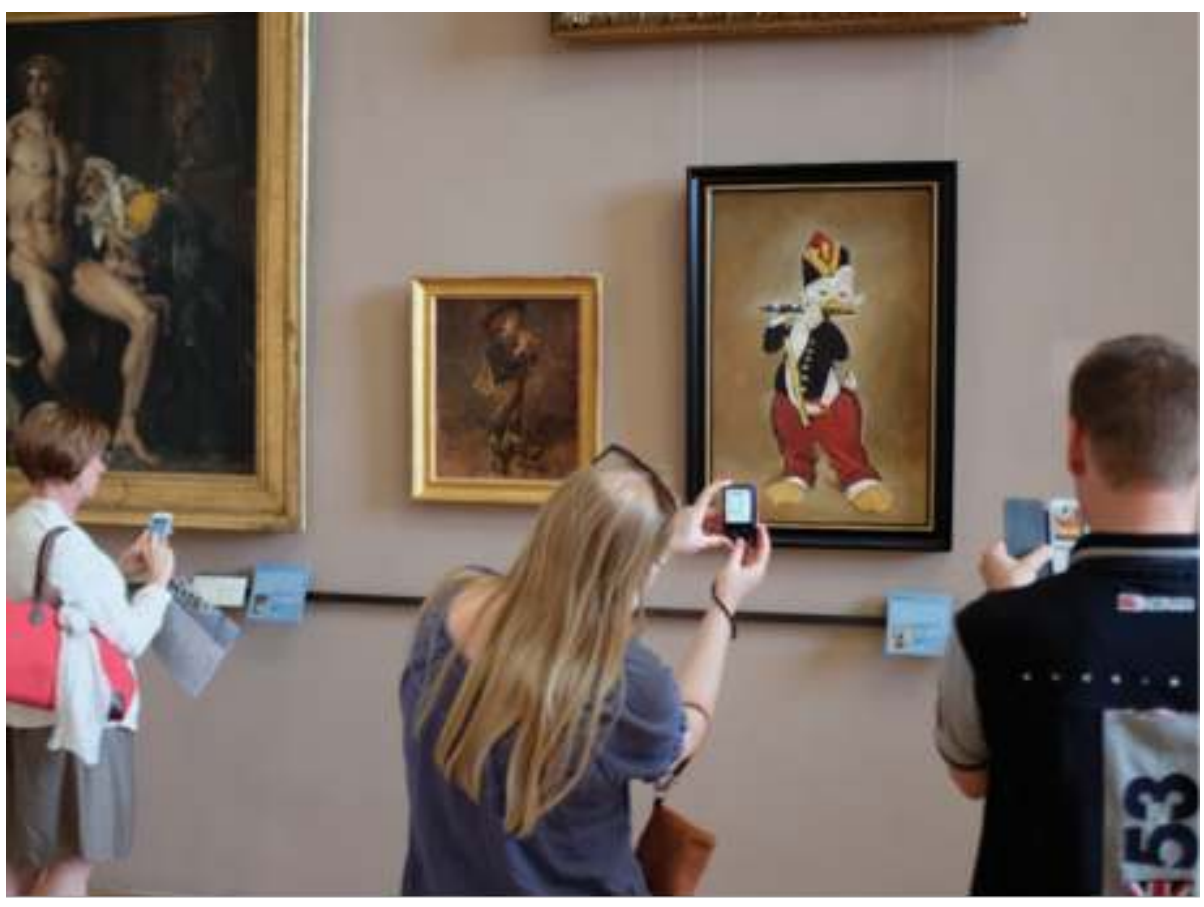

(c) Daniel Schmitt 
Le futur de la médiation numérique ? Mindy, le robot humanoöde du projet Corobot, dirigé par Patrice Caire, Université du Luxembourg. Photographié devant la Table pour le pendule de Foucault de Sophie Krier, Eppur si Muove, (MUDAM, Luxembourg, 2015).

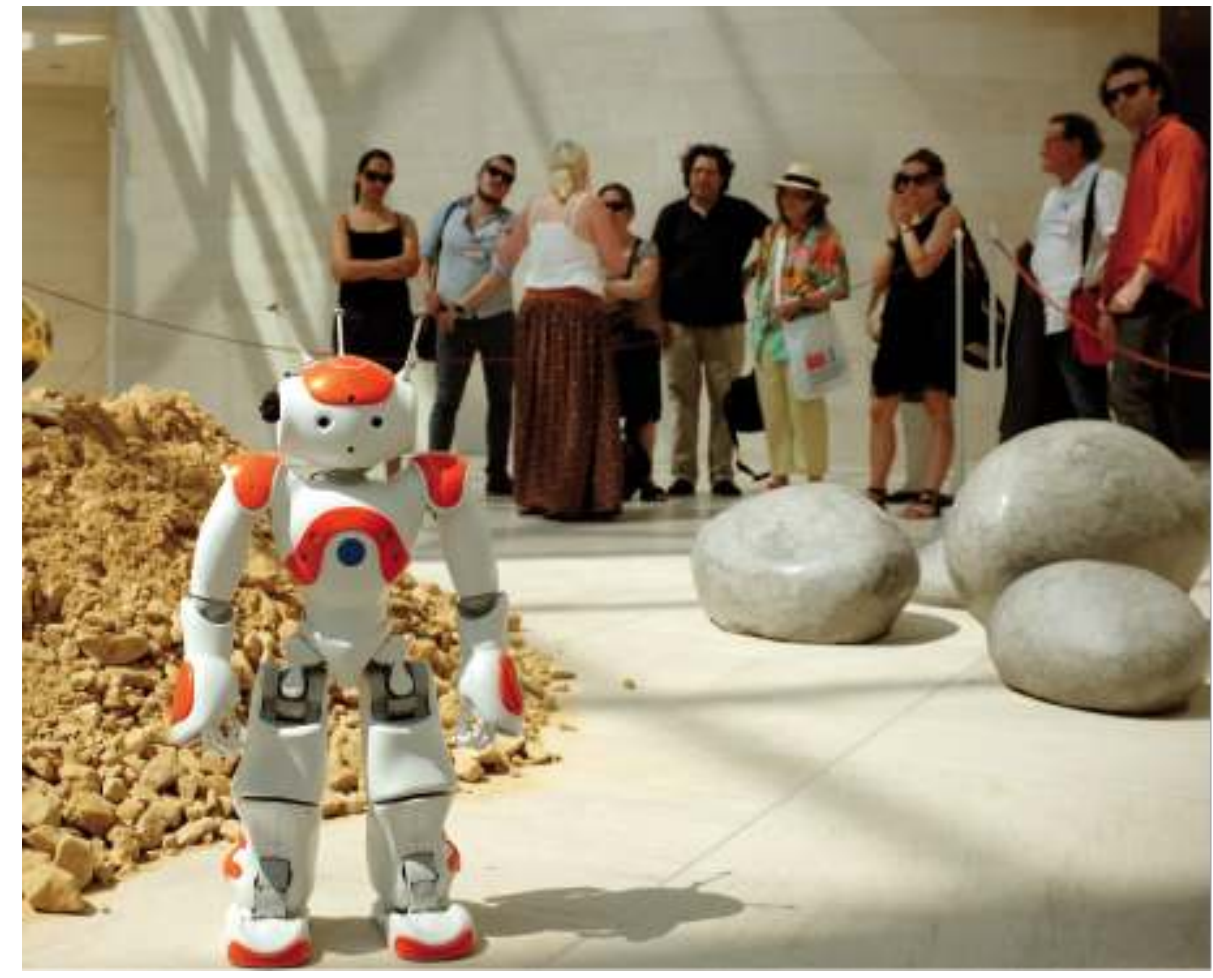

(C) Daniel Schmitt

\section{Le post-numérique : vers le numérique ordinaire}

En vingt ans, nous sommes passés d'une résistance aux écrans à un désir d'écrans dans l'exposition. Pour autant, cette tension n'a pas encore permis de développer et de stabiliser des nouvelles écritures. La médiation numérique est au musée ce que l'incunable était à l'imprimerie: un modèle ancien reproduit dans une technologie nouvelle ${ }^{3}$. On retrouve dans le numérique des pages de textes à feuilleter virtuellement ou à dérouler comme un volumen ou un rotulus du début de l'Histoire.

De façon invisible, les technologies numériques ont introduit des nouveaux acteurs (informatique, réseaux, design, ergonomie, sécurité...) dans le champ de la conception et modifié ceux de l'énonciation éditoriale ${ }^{4}$ : la parole du musée n'est plus le seul fait de l'institution. À partir d'un smartphone, des algorithmes peuvent, par exemple, construire des cartels automatiques en agrégeant des données issues du big data. La parole provient d'opérations calculatoires puisant dans l'ensemble dynamique des paroles anonymes du Web 2.0 (Toshniwal et al., 2015). Et finalement, cette parole provient de nulle part. Si dans l'expérience ordinaire cela n'est pas préjudiciable, dans l'expérience muséale, la parole dite est réputée « vraie ». C'est le point de vue du musée qui importe, celui qui inspire confiance aux visiteurs et qui leur sert à construire des liaisons robustes autour des œuvres et des expôts. C'est à la technologie de s'adapter aux besoins de médiation et non l'inverse. Les innovations technologiques offrent des opportunités pour inventer des nouvelles écritures qui ne soient pas un frein à la consultation, au fait de vivre une expérience singulière et collective. 
13 La médiation numérique permet de penser la médiation globale dans l'exposition non pas en tant que message à transmettre, mais comme un «bricolage » réussi d'objets, de paroles, d'images s'inscrivant dans la tension des attentes et des savoirs mobilisés par les visiteurs. À partir d'entretiens réalisés en re-situ subjectif, on peut identifier les principaux registres attentes-savoirs des visiteurs pour concevoir des nouvelles médiations (Schmitt et Meyer-Chemenska, 2014). Il s'agit aussi de prendre en compte l'ergonomie de l'apprentissage en situation naturelle de visite qui se différencie nettement $\mathrm{du}$ contexte scolaire. Cela signifie rendre la technologie davantage bienveillante pour les visiteurs, la rendre moins visible, plus ordinaire, moins fascinante, de façon à pouvoir penser et travailler une écologie de la médiation instrumentée. En ce sens, c'est bien le post-numérique dans les musées qu'il faut envisager aujourd'hui. Car c'est de l'effacement des interfaces par les usages dans le quotidien que viendra l'opportunité de penser ces dispositifs au service des visiteurs.

14 Le numérique constitue un défi majeur pour le musée car en 20 ans, il est devenu le lieu visible de l'innovation de la technologie numérique par excellence, tout à rebours de l'image du musée poussiéreux. Il s'agit bien pour l'institution muséale d'avoir une approche critique du numérique et d'en exiger qu'il se mette au service de son discours, de sa narration et de son point de vue sans lui déléguer la construction de sens. Cela signifie aussi s'opposer à l'idéologie fallacieuse de la naturalisation de la culture par les données et les algorithmes.

\section{BIBLIOGRAPHIE}

Andreacola, F. Musée et numérique, enjeux et mutations,

Revue française des sciences de l\&apos ;information et de la communication, $\mathrm{n}^{\circ} 5$ : http://

rfsic.revues.org/1056, 2014.

Bougenies, F., Houriez, J., Houriez, S. et Leleu-Merviel, S.

Musée pour tous. Un dispositif de découverte dans les murs

et son évaluation, Culture et Musées (à paraître 2015).

Gellereau, M., Lamboux-Durand, A., Brière, C., Da Lage, E., Fleury, C., Gawin, G., Gaillard, M. et Priégo, H. Projet «TEMICS » Témoignages et médiation interculturelle de collections du patrimoine sensible. Rapport final, université de Lille 3, ministère de la Culture et de la Communication, 2015.

Poizat, G. Le concept d'appropriation en formation des adultes, in Friedrich, J. et Pita Castro, J. Recherches en formation des adultes : un dialogue entre concepts et réalité.

Dijon : Raison \& Passions, 2014, pp. 40-68.

Schmitt, D. et Meyer-Chemenska, M. Expériences de visite :

de la transmission à la liction, La Lettre de l\&apos ;OCIM, n 155, 2014, pp. 17-23.

Souchier, E. et Jeanneret, Y. L\&apos ;énonciation éditoriale dans les écrits d\&apos ;écran,

Communication et langages, $\mathrm{n}^{\circ} 145,2005$, 
pp. 3-15.

Toshniwal, S., Sharma, P., Srivastava, S. et Sehgal, R. USHER : An Intelligent Tour Companion. IUI Companion \&apos ; 15 : Proceedings of the 20th International Conference on Intelligent User Interfaces Companion, 2015, pp. 81-84.

\section{NOTES}

1. Jusqu'au milieu des années 1990 , les musées diffusent essentiellement des films produits pour la télévision de sorte que l'écriture et le format sont rarement adaptés à une rediffusion dans les espaces d'exposition.

2. 1,3 million de visiteurs pour Cités-Cinés et près d'un million pour Mémoires d'Égypte (chiffres cumulés).

3. Nous devons cette formule à Denis Rohrer, conservateur, responsable des collections de 1\&apos;Alimentarium, Vevey, Suisse.

4. L'énonciation éditoriale "désigne l'ensemble de ce qui contribue à la production matérielle des formes qui donnent au texte sa consistance, son image de texte [...] Plus fondamentalement, l'énonciation éditoriale est ce par quoi le texte peut exister matériellement, socialement, culturellement... aux yeux du lecteur" (Souchier et Jeanneret, 2005).

\section{RÉSUMÉS}

À partir d'un rappel historique de l'usage du numérique dans les musées, les auteurs montrent comment les différents outils développés et leurs évolutions ont influé sur l'expérience de visite et comment une approche critique de la médiation numérique par les institutions muséales permet de penser la médiation globale de l'exposition.

\section{INDEX}

Mots-clés : numérique, musée

\section{AUTEURS}

\section{DANIEL SCHMITT}

maître de conférences à l'université de Valenciennes

daniel.schmitt@univ-valenciennes.fr

\section{MURIEL MEYER-CHEMENSKA}

muséographe et directrice de l'agence Métapraxis

muriel.meyer@metapraxis.fr 